\title{
A Comparison of Chemical Evolution between the Milky Way and M31 Galaxy
}

\author{
J. Yin ${ }^{1}$, J.L. Hou ${ }^{1}$, R.X. Chang ${ }^{1}$, S. Boissier ${ }^{2}$, and N. Prantzos ${ }^{3}$ \\ ${ }^{1}$ Shanghai Astronomical Observatory, CAS, Shanghai, 200030, China \\ email: jyin, houjl, crx@shao.ac.cn \\ ${ }^{2}$ Laboratoire d'Astrophysique de Marseillee, Traverse du Syphon-Les trois lucs BP8, \\ 13376 Marseille cedex 12, France email: samuel.boissier@oamp.fr \\ ${ }^{3}$ Institut d'Astrophysique de Paris, CNRS, 98bis Bd Arage, 75014, Paris, France \\ email: prantzos@iap.fr
}

\section{Motivation}

Andromeda galaxy (M31,NGC224) is the biggest spiral in the Local Group. By studying the star formation history(SFH) and chemical evolution of M31, and comparing with the Milky Way Galaxy, we are able to understand more about the formation and evolution of spiral galaxies.

\section{Method and main results}

Firstly, we apply the same parameters for the chemical evolution model that well fit the Milky Way disk to the M31 disk evolution. It is found that the obtained radial profiles of gas surface density and star formation rate (SFR) have great difference from the observed results in M31 disk. If the Schmidt type SFR law is valid for both disks, then, the corresponding star formation efficiency (SFE) must be greatly different between these two galaxy disks. Then, we use the observed gas and SFR profiles of M31 to determine the SFE in M31 disk by assuming Schmidt type SFR. It appears that SFE in M31 disk should be proportional to $1 / \mathrm{r}$. Based on this new SFR, our best model shows that the total mass in M31 disk should be $(3-4) 10^{10} M_{\odot}$, and radial dependent of infall time scale is about $0.2 \mathrm{r}+1 \mathrm{Gyr}$, more flat than in the Milky Way disk. The best model can roughly reproduce the abundance features along the disk. But there are some problems with this kind of model. For example, the predicted gas fraction is much higher compared with the value in the Milky Way, the inner disk has high element abundances. All of these problems should be relate to the form how SFR depend on radius. It is a common knowledge that the radius dependence of infall time scale is the main mechanism of the existence of disk abundance gradient. But through our work we find that the behavior of SFR profile (how it depends on radius) can also significantly influence the abundance gradient.

Comparing with Galaxy, M31 has much lower gas and SFR profiles, but their total dynamic mass are comparable. That means M31 needs faster star formation rate and infall rate (namely shorter infall time scale) in order to consume more gas. However, the low metallicity and flat abundance gradient require lower star formation efficiency, and few metal poor stars means the infall time scale can not be very short. The chemical evolution model of the Milky Way disk can not fit the M31's features. They must have very different SFH. Indeed, there exists a lot of obvious evident that M31 had some interaction or accretion with companions in the past time. Those processes must play important roles in the formation and evolution of the M31 galaxy. 